\title{
Synthesis of I-3, 11-3-bis-(methylthio)biflavones from the corresponding bichalcones: a new application of the $\mathrm{I}_{2}-\mathrm{Me}_{2} \mathrm{SO}-\mathrm{H}_{2} \mathrm{SO}_{4}$ reagent system
}

\author{
Jawaid Iqbal, ${ }^{\text {a,* }}$ Anamika Gupta, ${ }^{\text {a }}$ and Khwaja Ishratullah ${ }^{b}$ \\ ${ }^{a}$ Organic Chemistry Section, Department of Chemistry, Aligarh Muslim University, Aligarh- \\ 202002, India \\ ${ }^{\mathrm{b}}$ Organic Chemistry Division-II, Indian Institute of Chemical Technology, Hyderabad-500007, \\ India \\ E-mail: Jawaid.Iqbal0@lycos.com
}

\begin{abstract}
The synthesis is described of I-3, II-3-bis-(methylthio)-I-4', II-4', I-5, II-5, I-7, II-7hexamethoxy-[1-3', II-8]-biflavone (2) and I-3, II-3-bis-(methylthio)-I-4', II-4', I-5, II-5, I-7, II-7hexamethoxy-[I-3', II-6]-biflavone (4), in one step from bichalcones $\mathbf{1}$ and $\mathbf{3}$, respectively, using the $\mathrm{I}_{2}-\mathrm{Me}_{2} \mathrm{SO}-\mathrm{H}_{2} \mathrm{SO}_{4}$ reagent system.
\end{abstract}

Keywords: Biflavonoid, bichalcone, bis-(methylthio)biflavone, dimethyl sulfoxide

\section{Introduction}

The oxidation-reduction cycle, ${ }^{1}$ that sets in when dimethyl sulfoxide is reduced to dimethyl sulfide in the presence of a catalytic amount of halogen, has found potential synthetic applications. ${ }^{1-4}$ Furukawa et al. ${ }^{1}$ have used the halogen-dimethyl sulfoxide-sulfuric acid system as an oxidizing reagent for active aldehydes and ketones. Previous reports from our laboratory described its applications in the high-yield dehydrogenation of the 2,3-dihydropyrone ring of various flavanoid systems ${ }^{2,3}$ and in a one-step synthesis of 3-iodoflavones from 2'hydroxychalcones. ${ }^{4}$ Furukawa's report indicated that in the oxidation of some active methylene ketones with this reagent system, the diketone oxidation product was accompanied by substantial amount of $\alpha$-methylthio-ketone, when the reaction was carried under sealed tube conditions, i.e., when the in situ-generated dimethyl sulfide was allowed to react with the initially formed $\alpha$ iodoketone. ${ }^{1}$ Within the context of our work aimed at the synthesis and determination of absolute stereochemistry of biflavanoids, we studied the reaction of some 2',2"'-dihydroxybichalcones with $\mathrm{I}_{2}-\mathrm{Me}_{2} \mathrm{SO}-\mathrm{H}_{2} \mathrm{SO}_{4}$ under sealed-tube conditions, and now report a new application of this reagent system in the synthesis of the hitherto unknown I-3, II-3-bis-(methylthio)biflavone. 


\section{Results and Discussion}

2',2'"-Dihydroxy-4,4',4",4"',6',6"'-hexamethoxy-[3,3'"]-bichalcone (1) was first heated at $100^{\circ} \mathrm{C}$ for $c a .15$ min. with dimethyl sulfoxide and a small amount of sulfuric acid, then a catalytic amount of iodine was added, and the mixture was heated in a sealed tube at $100^{\circ} \mathrm{C}$ for $2 \mathrm{~h}$. The major product, obtained in 63\% yields after workup and chromatographic purification, was characterized as I-3, II-3-bis-(methylthio)-I-4', II-4', I-5, II-5, I-7, II-7-hexamethoxy-[I-3', II-8]biflavone (2), m.p. $198^{\circ} \mathrm{C}$, from its UV, ${ }^{1} \mathrm{H}$ - and ${ }^{13} \mathrm{C}$ - NMR. and mass spectra (Scheme 1).

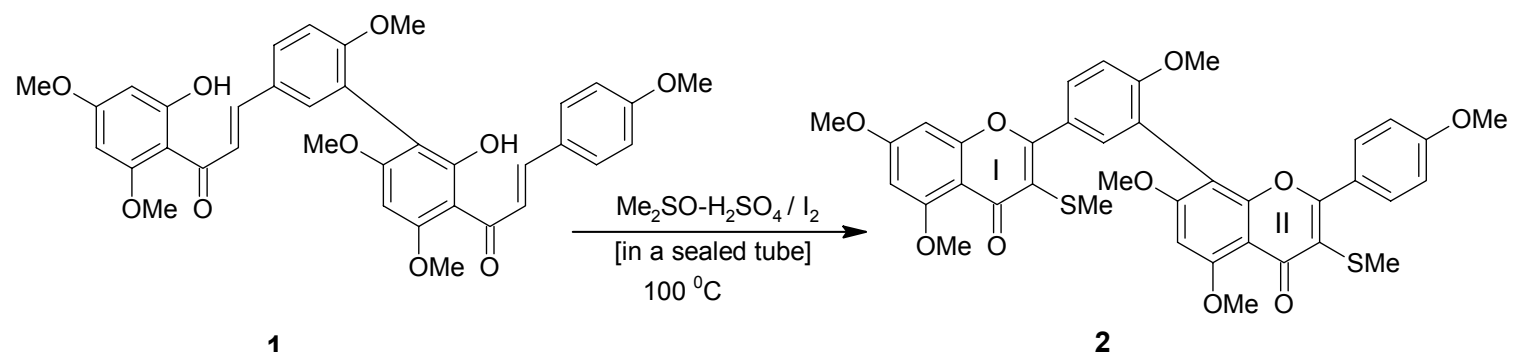

\section{Scheme 1}

The ${ }^{1} \mathrm{H}$ - NMR spectrum of $\mathbf{2}$ indicated the intact presence of all the aromatic ring protons and the absence of $\alpha$ - and $\beta$ - protons of bichalcone. Further, two singlets at $\delta 2.91$ and 2.94 each integrating for three protons indicated the presence of two methylthio $\left(\mathrm{CH}_{3}-\mathrm{S}-\right)$ groups at I-C-3 and II-C-3. The ${ }^{13} \mathrm{C}$ - NMR spectral data are also in complete accord with the assigned structure. The mass spectrum shows the molecular ion peak at $\mathrm{m} / \mathrm{z} 714$ (40\%), and retro- Diels-Alder fragments at $\mathrm{m} / \mathrm{z} 537,357,223,181$ and 178 as required by structure 2

The formation of bis-(methylthio)biflavone from bichalcone appears to be general. When this reaction was extended with 2',2"'-dihydroxy-4,4',4",4"',6',6"'- hexamethoxy-[3,5"']-bichalcone (3) under similar condition, it completed in $1.5 \mathrm{~h}$, and on usual workup and purification afforded I-3, II-3-bis-(methylthio)-I-4',II-4',I-5,II-5,I-7,II-7-hexamethoxy-[I-3',II-6]-biflavone (4), in 60\% yield (Scheme 2). The observed spectral data, UV, ${ }^{1} \mathrm{H}-$ and ${ }^{13} \mathrm{C}-\mathrm{NMR}$ are compatible with the assigned structure.

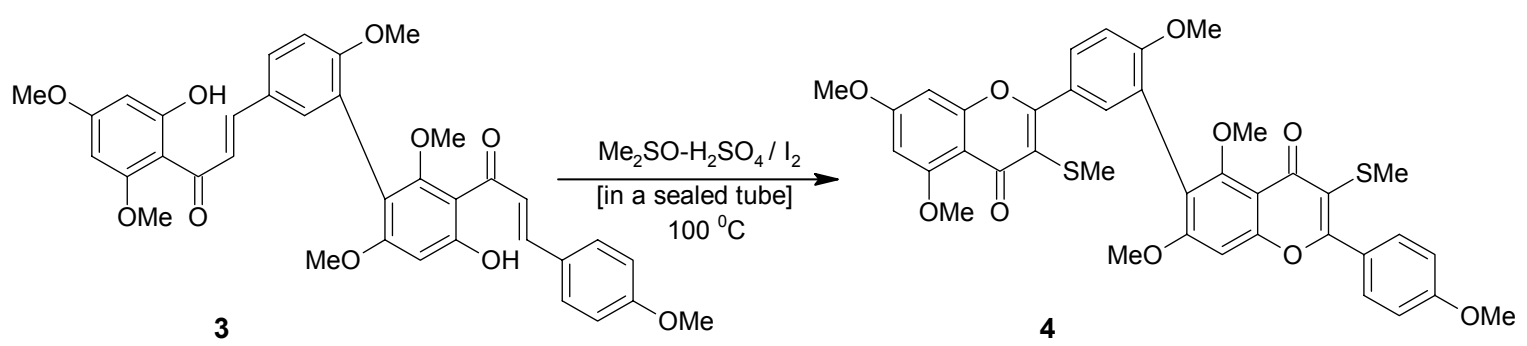

Scheme 2 
Further extension with 2'-hydroxy-4,4',6'-trimethoxychalcone (5), yielded 3-methylthio$5,7,4^{\prime}$-trimethoxyflavone (6), m.p. $150^{\circ} \mathrm{C}$, as major product, characterized from its UV, NMR and mass spectra.

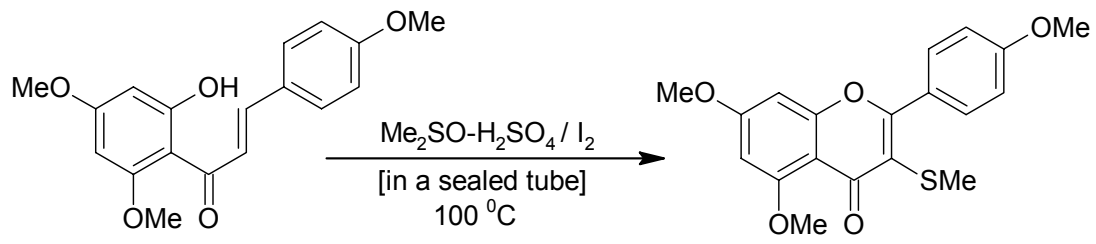

\section{Scheme 3}

This, a one step transformation of bis-(2'-hydroxy)bichalcone to bis-(methylthio)biflavone with iodine-dimethyl sulfoxide-sulfuric acid reagent system is interesting both from mechanistic and synthetic viewpoints. Introduction of a sulfur- containing substituent $\alpha$ - to a carbonyl group is often a key step in organic synthesis. ${ }^{5}$ Although recent advances have removed some of the difficulties associated with the method of sulfenylation of aldehydes and ketones, ${ }^{6}$ the convenience and mildness of this method may provide further improvement. In this context it may be mentioned that 3-sulfinylchromone was synthesized by base-induced acylation of sulfinyl ketone. ${ }^{7}$

A reasonable mechanism for these reactions is that shown in Scheme 4. The starting chalcone isomerises ${ }^{4}$ in the presence of conc. sulfuric acid to the corresponding flavanone 7 , which undergoes iodination at $\mathrm{C}-3$ to give isomeric 3-iodoflavanones. ${ }^{2-4}$ In the light of our previous experiments $^{2-4}$, we argue that the C-3- iodination of flavanone produces two isomeric 3iodoflavanones, 8 and $\mathbf{9}$. In the present conditions $\mathbf{8}$ is formed predominantly and has an unfavorable conformation for trans- diaxial dehydrohalogenation. The differential predominance of the isomeric 3-iodoflavanones $\mathbf{8}$ or $\mathbf{9}$, starting from chalcone or flavanone, respectively, have been observed and discussed in our previous reports ${ }^{3,4}$ and is probably related with kinetic versus thermodynamic control: however, the way in which the chalcone-flavanone equilibrium affects this differential formation of 3-iodoflavanones is yet unclear. The major product, methylthioflavone was accompanied by a very small amount of the corresponding flavone 10 and 3-iodoflavone 11. However, the formation of flavonol 12, which could have resulted from an oxidation-reduction cycle, ${ }^{1}$ was not observed in the experiments described. 


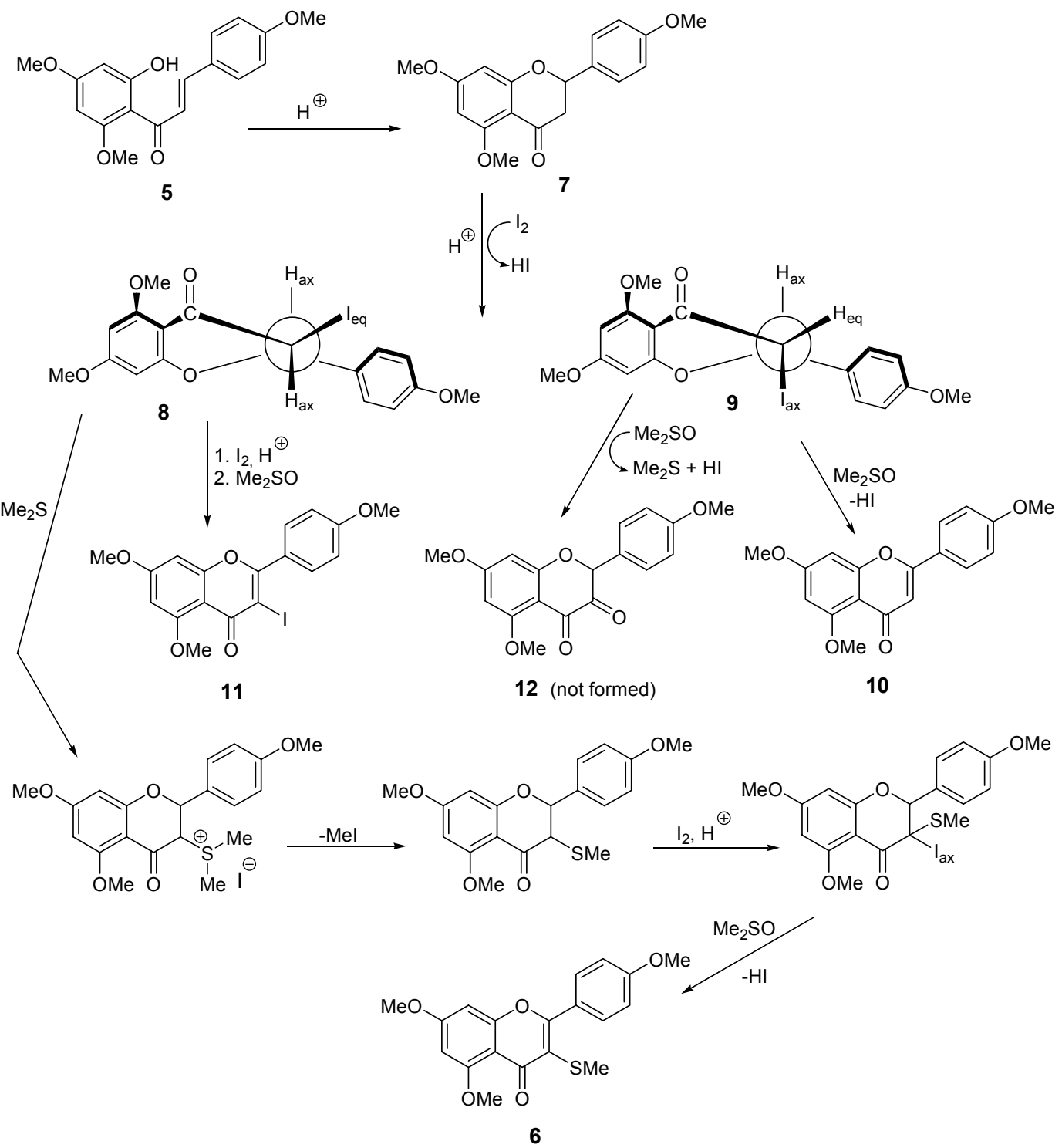

\section{Scheme 4}

\section{Experimental Section}

General Procedures. M.p.s were taken on a Kofler block and are uncorrected, ${ }^{1} \mathrm{H}-\mathrm{NMR}$ spectra were recorded on Varian A-60D and JEOL 4H-100 instruments and ${ }^{13} \mathrm{C}-\mathrm{NMR}$ spectra with JEOL FX-100 spectrometer with $\mathrm{Me}_{4} \mathrm{Si}$ as internal standard. Mass spectra were obtained on a JEOL-OSIG mass spectrometer at $75 \mathrm{eV}$. Commercial iodine was used without further purification. Dimethyl sulfoxide was dried by distillation from calcium hydride under reduced pressure. 
Reaction of $2^{\prime}, 2^{\prime \prime \prime}$-dihydroxy-4,4',4"',4"',6',6"'-hexamethoxy[3,3'"']-bichalcone (1) with $I_{2}-$ $\mathrm{Me}_{2} \mathrm{SO}-\mathrm{H}_{2} \mathrm{SO}_{4}$ reagent system. A mixture of $1(630 \mathrm{mg}, 1.0 \mathrm{mmol})$ and sulfuric acid $(60 \mathrm{mg}$, $0.6 \mathrm{mmol})$ in $\mathrm{Me}_{2} \mathrm{SO}(5 \mathrm{ml})$ was first heated at $100^{\circ} \mathrm{C}$ for $c a .15 \mathrm{~min}$., then cooled to room temperature. After adding iodine $(50 \mathrm{mg}, 0.2 \mathrm{mmol})$, the mixture was further heated in a sealed tube at $100^{\circ} \mathrm{C}$ for $\sim 2 \mathrm{~h}$. It was then poured into ice-water and the precipitate was filtered, washed with water and dried to give a solid which was chromatographed on silica gel $(25 \mathrm{gm})$. Elution with chloroform-n-hexane (60:40) gave (450 mg, 63\%) of I-3, II-3-bis-(methylthio)-I-4', II-4', I5, II-5, I-7, II-7- hexamethoxy [I-3',II-8]biflavone (2), m.p. $198^{\circ} \mathrm{C}$ (Found: C, 63.7; H, 4.8; $\mathrm{C}_{38} \mathrm{H}_{34} \mathrm{O}_{10} \mathrm{~S}_{2}$ requires: $\left.\mathrm{C}, 63.8 ; \mathrm{H}, 4.7 \%\right) ; \lambda_{\max }(\mathrm{MeOH}) 264,341 \mathrm{~nm} ;{ }^{1} \mathrm{H}-\mathrm{NMR}: \delta_{\mathrm{H}}\left(\mathrm{CDCl}_{3}\right)$ : $2.91\left(3 \mathrm{H}, \mathrm{s},-\mathrm{S}-\mathrm{CH}_{3}\right), 2.94\left(3 \mathrm{H}, \mathrm{s},-\mathrm{S}-\mathrm{CH}_{3}\right), 3.75(6 \mathrm{H}, \mathrm{s}, \mathrm{OMe}-\mathrm{I}-7, \mathrm{II}-7), 3.82$ (3H, s, OMe-II4 ),3.87 (3H,s,OMe-I-4),3.92 (3H,s,OMe-I-5), 4.07 (3H, s, OMe-II-5), 6.32 (1H, d, J=2.5 Hz, HI-6), 6.47 (1H, d, J=2.5 Hz, H-I-8), 6.63 (1H, s, H-II-6), 6.76 (2H, d, J=8.5 Hz, H-II-3', 5'), 7.11 (1H, s, H-I-5'), 7.38 (2H, d, J=8.5 Hz, H-II-2',6'), 7.89 (1H, dd, J=2.5 Hz, J2=8.5 Hz, H-I-6'), 7.81 $(1 \mathrm{H}, \mathrm{s}, \mathrm{H}-\mathrm{I}-2 \mathrm{\prime})$; ${ }^{13} \mathrm{C}-\mathrm{NMR}: \delta_{\mathrm{C}}\left(\mathrm{CDCI}_{3}\right)$ 23.1, $23.4\left(-\mathrm{S}-\mathrm{CH}_{3}\right), 55.6$ (I, II-4' OMe), $56 . \mathrm{O}$ (I, II-7OMe), 56.2, 56.4 (I, II-5-OMe), 92.7 (I-C-8), 95.4 (II-C-6), 98.0 (I-C-6), 103.1, 103.9, 104.0, 104.2 (I, II-C-3, II-C-8, I, II-C-10), 111.4 (II-C-3', 5'), 114.5 (I-C-5'), 121.2, 122.6 (I-C-3', I, IIC-1'), 127.8 (I-C-2',6 ${ }^{1}$ ), 128.3 (II-C-2'), 130.8 (II-C-6'), 153.5 (II-C-9), 157.7 (I-C-9), 160.4, 161.1, 161.4 (I, II-C-5, I-C-4', II-C-7), 162.7 (II-C-4'), 163.5 (I, II-C-2), 165.6 (I-C-7), 181.9, 182.2 (I, II-C-4); m/z $714\left(40, \mathrm{M}^{+}\right.$), 668, 537, 534, 491, 483, 357, 181, (100), 178, (Found M+, 714.8161, Calcd. for $\left.\mathrm{C}_{38} \mathrm{H}_{34} \mathrm{O}_{10} \mathrm{~S}_{2}: \mathrm{M}_{\mathrm{r}}, 714.8166\right)$.

Reaction of $2^{\prime}, 2^{\prime \prime \prime}$-dihydroxy-4,4',4",4"', $6^{\prime}, 6^{\prime \prime}-$ hexamethoxy-[3, 5'"']-bichalcone (3) with $I_{2}-$ $\mathrm{Me}_{2} \mathrm{SO}-\mathrm{H}_{2} \mathrm{SO}_{4}$ reagent system. Reaction as above, using $3(630 \mathrm{mg}, 1.0 \mathrm{mmol}), \mathrm{H}_{2} \mathrm{SO}_{4}(50 \mathrm{mg}$, $0.5 \mathrm{mmol})$ and $\mathrm{I}_{2}(50 \mathrm{mg}, 0.2 \mathrm{mmol})$ in $4.5 \mathrm{ml}$ of $\mathrm{Me}_{2} \mathrm{SO}$ at $100^{\circ} \mathrm{C}$ for $\sim 1.5 \mathrm{~h}$, gave, after usual work-up and chromatographic purification (silica gel), (400 mg, 60\%) of I-3, II-3-bis (methylthio)-I-4',II-4 ${ }^{1}$, I-5, II-5, I-7, II-7-hexamethoxy[1-3', II-6]-biflavone (4), crystallized from acetone-methanol, m.p. $233^{\circ} \mathrm{C}$. (Found: $\mathrm{C}, 63.2 ; \mathrm{H}, 4.9 ; \mathrm{C}_{38} \mathrm{H}_{34} \mathrm{O}_{10} \mathrm{~S}_{2}$ requires: C, 63.8; H, 4.7\%); $\lambda_{\max }(\mathrm{MeOH}) 263,343 \mathrm{~nm} ;{ }^{1} \mathrm{H}-\mathrm{NMR}: \delta_{\mathrm{H}}\left(\mathrm{CDCI}_{3}\right) 2.90\left(3 \mathrm{H}, \mathrm{s},-\mathrm{S}-\mathrm{CH}_{3}\right), 2.92\left(3 \mathrm{H}, \mathrm{s},-\mathrm{S}-\mathrm{CH}_{3}\right)$, 3.61, 3.86,3.87, 3.88, 3.91, 3.92 (3H each, s, OMe -II-5', II-4', II-7, I-7, I-4', I-5), 6.33 (1H, d, $\mathrm{J}=2.5 \mathrm{~Hz}, \mathrm{H}-\mathrm{I}-6), 6.56(1 \mathrm{H}, \mathrm{d}, \mathrm{J}=2.5 \mathrm{~Hz}, \mathrm{H}-\mathrm{I}-8), 6.79$ (1H, s, H-II-8), 7.03 (2H, d, J=8.7 Hz, H-II$\left.3^{\prime}, 5^{\prime}\right), 7.08\left(1 \mathrm{H}, \mathrm{d}, \mathrm{J}=8.7 \mathrm{~Hz}, \mathrm{H}-\mathrm{I}-5^{\prime}\right), 7.61$ (2H, d, J=8.7 Hz, H-II-2', 6'), 7.80 (1H, d, J=2.5 Hz, H-I-2'), $7.86\left(1 \mathrm{H}, \mathrm{dd}, \mathrm{J}_{1}=2.5 \mathrm{~Hz}, \mathrm{~J}_{2}=8.7 \mathrm{~Hz}, \mathrm{H}-\mathrm{I}-6{ }^{\prime}\right) ;{ }^{13} \mathrm{C}-\mathrm{NMR}: \delta_{\mathrm{C}}\left(\mathrm{CDCI}_{3}\right) 23.3,23.6\left(-\mathrm{S}_{-} \mathrm{CH}_{3}\right)$, 55.6 (I, II-4'-OMe), 56.1 (I, II-7-OMe), 56.3, 56.5 (I, II-5-OMe), 93.8, 94.4 (I, II-C -8), 95.4 (IC-6), 102.9, 103.2, 103.9, 104.1, 104.3 (I, II-C-3, II-C-6, I, II-C-10), 114.1 (II-C-3', 5'), 115.9 (IC-5'), 121.1 (I-C-3'), 122.4 (I, II-C-1'), 127.2, 128.3, 130.6 (I, II-C -2', 6'), 157.6 (I, II-C-9), 159.1 (II-C-5), 160.4, 161.1, 161.5 (I, II -C-4', I-C-5), 163.1, 163.7, 164.1 (I, II-C-7, 2), 181.7, 181.9 (I, II-C-4); m/z $714\left(31, \mathrm{M}^{+}\right.$), 537, 536, 534, 491, 483, 477, 357, 181 (100), 135, (Found $\mathrm{M}^{+}, 714.8159$, Calcd. For $\left.\mathrm{C}_{38} \mathrm{H}_{34} \mathrm{O}_{10} \mathrm{~S}_{2}: \mathrm{M}_{\mathrm{r}}, 714.8166\right)$.

Reaction of 2'-hydroxy-4, 4', 6'-trimethoxychalcone (5) with $\mathrm{I}_{2}-\mathrm{Me}_{2} \mathrm{SO}-\mathrm{H}_{2} \mathrm{SO}_{4}$ reagent system. The reaction, according to the above procedure, using 5 (470 mg, $1.5 \mathrm{mmol}), \mathrm{H}_{2} \mathrm{SO}_{4}(40$ $\mathrm{mg}, 0.4 \mathrm{mmol})$ and $\mathrm{I}_{2}(50 \mathrm{mg}, 0.2 \mathrm{mmol})$ in $4 \mathrm{ml}$ of $\mathrm{Me}_{2} \mathrm{SO}$ at $100^{\circ} \mathrm{C}$ for $1.5 \mathrm{~h}$, gave, after the 
usual work-up and chromatographic purification (silica gel), (350 mg, 65\%) of 3-methylthio5,7,4'-trimethoxyflavone (6), m.p. $150^{\circ} \mathrm{C}$, as major product; $\lambda_{\max }(\mathrm{MeOH}) 262,341 \mathrm{~nm} ;{ }^{1} \mathrm{H}-$ NMR: $\delta_{\mathrm{H}}\left(\mathrm{CDCI}_{3}\right) 2.89\left(3 \mathrm{H}, \mathrm{s},-\mathrm{S}-\mathrm{CH}_{3}\right), 3.90(3 \mathrm{H}, \mathrm{s}, \mathrm{OMe}-4), 3.97(6 \mathrm{H}, \mathrm{s}, \mathrm{OMe}-5,7), 6.46(1 \mathrm{H}$, s, H-6), 6.65 (1H, s, H-8), $7.06\left(2 \mathrm{H}, \mathrm{d}, \mathrm{J}=8.6 \mathrm{~Hz}, \mathrm{H}-33^{\prime}, 5^{\prime}\right), 8.04\left(2 \mathrm{H}, \mathrm{d}, \mathrm{J}=8.6 \mathrm{~Hz}, \mathrm{H}-2^{\prime}, 66^{\prime}\right) ;{ }^{13} \mathrm{C}-$ NMR: $\delta_{\mathrm{C}}\left(\mathrm{CDCl}_{3}\right) 22.6\left(-\mathrm{S}_{-} \mathrm{CH}_{3}\right), 55.8,56.2,56.5,\left(5,7,44^{\prime}-\mathrm{OMe}\right), 94.1(\mathrm{C}-6), 98.6(\mathrm{C}-8), 103.6$ (C-3), 109.1 (C-10), 121.2 (C-3', 5'), 127.9 (C-1'), 130.1 (C-2', 6'), 157.1 (C-9), 161.1,161.3 (C-

2,5), 161.9 (C-4'), 163.8 (C-7), 181.6 (C-4); m/z 358 (41, M+), 331, 223, 181 (100), 178, 135 (Found $\mathrm{M}^{+}, 358.4155$, Calcd. for $\mathrm{C}_{19} \mathrm{H}_{18} \mathrm{O}_{5} \mathrm{~S}: \mathrm{M}_{\mathrm{r}}, 358.4163$ ).

\section{References}

1. Furukawa, N.; Akasaka, T; Aida, T; Oae, S. J. Chem. Soc., Perkin Trans. 1 1977, 372.

2. Fatma, W.; Iqbal, J.; Ismail, H.; Ishratullah, Kh.; Shaida, W.A.; Rahman, W. Chem. Ind. (London) 1979, 315.

3. Fatma, W.; Iqbal, J.; Manchanda, V.; Shaida, W.A.; Rahman, W. J. Chem. Research, 1984 (S) 298; (M) 2656.

4. Iqbal, J.; Fatma, W.; Shaida, W.A.; Rahman, W., J. Chem. Research 1982, (S) 92; 1001.

5. Trost, B. M. Acc. Chem. Res. 1978, 11, 453.

6. House, H. Modern Synthetic Reactions, W.A. Benjamin, Menlo Park, California, 1972.

7. Von Strandtmann, M.; Klutchko, S.; Cohen, M. P.; Shavvel, J. J. Heterocycl. Chem. 1974, 11, 183. 